Results 63 pts were randomized to TLPO (n=43) vs TLPLDC $(n=20)$. The TLPO cohort contained more females and received less chemotherapy ( $0 \%$ vs $10 \%)$, but otherwise were comparable. There were no differences in DFS $(p=0.948)$ or OS $(p=0.779)$ between the two vaccines (figures 1\&2). Comparing the TLPO pts to all other pts in the phase IIb trial [TLPLDC+G-CSF $(n=57)$, TLPLDC-G-CSF $(n=46)$, and placebo $(n=41)]$ the TLPO arm had improved DFS compared to placebo $(p=0.019)$ and TLPLDC+G-CSF $(p=0.001)$, but roughly equivalent to the TLPLDC-G-CSF arm $(p=0.276)$ (figure 3). A similar trend was seen in OS analysis, though differences were not statistically significant (figure 4).

Conclusions TLPO and TLPLDC vaccines (without the use of G-CSF) improve DFS in patients with resected stage III/IV melanoma compared to placebo. The TLPO vaccine may offer advantages via reduced cost and vaccine production time. TLPO should be closely considered for further clinical trials.

Trial Registration NCT02301611: Phase IIB TL + YWCP + DC in MelanomaTLPLDC IND\#16101TLPO IND\#17274

Ethics Approval This study was approved by WIRB; protocol \#20141932

http://dx.doi.org/10.1136/jitc-2020-SITC2020.0431

\section{3-YEAR RESULTS OF THE PHASE 2 RANDOMIZED TRIAL FOR TALIMOGENE LAHERPAREPVEC (T-VEC) NEOADJUVANT TREATMENT PLUS SURGERY VS SURGERY IN PATIENTS WITH RESECTABLE STAGE IIIB- IVM1A MELANOMA}

${ }^{1}$ Reinhard Dummer*, 2David Gyorki, ${ }^{3}$ ohn Hyngstrom, ${ }^{4}$ Adam Berger, ${ }^{5}$ Robert Cony, ${ }^{6}$ Lev Demidov, ${ }^{7}$ Edward Chan, ${ }^{7}$ Hoi-Shen Radcliffe, ${ }^{8}$ Mark Faries, ${ }^{9}$ Merrick Ross. ${ }^{1}$ University Hospital of Zurich, Zurich, SC, Switzerland; '2Peter MacCallum Cancer Centre, Melbourne, Australia; ${ }^{3}$ University of Utah Huntsman Cancer Insti, Salt Lake City, UT, USA; ${ }^{4}$ Cancer Institute of New Jersey, New Brunswick, NJ, USA; ${ }^{5}$ University of Alabama School of Medicine, Birmingham, AL, USA; ${ }^{6}$ N.N. Blokhin Russian Cancer Research Cen, Moscow, Russian Federation; ${ }^{7}$ Amgen Inc., Thousand Oaks, CA, USA; ${ }^{8}$ The Angeles Clinic and Research Institu, Los Angeles, CA, USA; ${ }^{9}$ University of Texas MD Anderson Cancer $C$, Houston, TX, USA

Background Neoadjuvant immunotherapies and targeted therapies for advanced melanoma are an active area of investigation. This is the first clinical trial of an approved oncolytic viral immunotherapy as a neoadjuvant treatment in advanced melanoma and the largest randomized controlled neoadjuvant trial including all types of resectable regional metastases to date. Previously published 2-year primary analysis results reported improved recurrence-free survival (RFS, HR 0.66, $\mathrm{P}=0.038$ ) and overall survival (OS, HR $0.49, \mathrm{P}=0.050$ ) for neoadjuvant $\mathrm{T}-\mathrm{VEC}$ plus surgery vs immediate surgery in resectable stage IIIB-IVM1a melanoma patients. $^{1}$ Here, we report the 3 -year interim analysis results.

Methods Patients with resectable stage IIIB-IVM1a melanoma and $\geq 1$ injectable cutaneous, subcutaneous, or nodal lesions were randomized $1: 1$ to receive 6 doses/12 weeks of neoadjuvant T-VEC then surgery (Arm 1) vs immediate surgical resection (Arm 2). T-VEC was administered until surgery, no remaining injectable tumors, or intolerance. RFS was defined as time from randomization to the first of local, regional, or distant recurrence, or death, where patients who did not receive surgery were imputed as events at baseline. Key secondary and exploratory endpoints include safety, an RFS sensitivity analysis that censored events at the start of subsequent anticancer therapy, OS, and eventfree survival (EFS), defined as time from randomization to disease progression that precludes surgery, or local, regional or distant recurrence post-surgery, or death from any cause, whichever occurs first. All $\mathrm{P}$ values are descriptive. NCT02211131.

Results As of April 30, 2020, median follow-up for all patients was 41.3 months. For Arm 1 vs. Arm 2, the 3-year KM estimates of RFS were $46.5 \%$ vs. $31.0 \%$ (HR 0.67 , $\mathrm{P}=0.043)$. In the RFS sensitivity analysis that removed the potential effect of subsequent anticancer therapy on RFS, the 3-year Kaplan-Meier (KM) estimates of RFS were $49.1 \%$ for Arm 1 and $22.9 \%$ for Arm 2 (HR 0.60, $\mathrm{P}=0.022$ ). The 3 -year $\mathrm{KM}$ estimates of EFS were $50.3 \%$ for Arm 1 and 32.7\% for Arm 2 (HR 0.58, P=0.015). For OS, the 3-year KM estimates were 83.2\% for Arm 1 and $71.6 \%$ for Arm 2 (HR 0.54, P=0.061). No new safety signals were detected.

Conclusions At 3-year follow up, we continued to observe improved RFS and OS and observed improved EFS with neoadjuvant T-VEC plus surgery compared with surgery alone. These results build upon the prior 2-year results to support the treatment effect of neoadjuvant T-VEC on advanced resectable melanoma. The final analysis will occur at 5 years.

Acknowledgements - The authors thank the investigators, patients, and study staff who are contributing to this study. The study was sponsored and funded by Amgen Inc. - Medical writing support was provided by Christopher Nosala (Amgen Inc.).

Trial Registration NCT02211131

Ethics Approval The study was approved by all institutional ethics boards.

\section{REFERENCE}

1. Dummer R, Gyorki DE, Hyngstrom J, et al. Primary 2-year (yr) results of a phase II, multicenter, randomized, open-label trial of efficacy and safety for talimogene laherparepvec (T-VEC) neoadjuvant (neo) treatment (tx) plus surgery (surg) vs surg in patients (pts) with resectable stage IIIB-IVM1a melanoma. Ann Oncol 2019;30 v903.

http://dx.doi.org/10.1136/jitc-2020-SITC2020.0432

\section{TALIMOGENE LAHERPAREPVEC (T-VEC) IN COMBINATION WITH IPILIMUMAB (IPI) VERSUS IPI ALONE FOR ADVANCED MELANOMA: 4-YEAR INTERIM ANALYSIS OF A RANDOMIZED, OPEN-LABEL, PHASE 2 TRIAL}

${ }^{1}$ Igor Puzanov*, ${ }^{2} J a s o n$ Chesney, ${ }^{3}$ Frances Collichio, ${ }^{4}$ Parminder Singh, ${ }^{5}$ Mohammed Milhem, ${ }^{6}$ John Glaspy, ${ }^{7}$ Omid Hamid, ${ }^{8}$ Merrick Ross, ${ }^{9}$ Philip Friedlander, ${ }^{10}$ Claus Garbe, ${ }^{11}$ Theodore Logan, ${ }^{12}$ Axel Hauschild, ${ }^{13}$ Celeste Lebbe, ${ }^{14}$ Min Yi, ${ }^{14}$ Wendy Snyder, ${ }^{15}$ Janice Mehnert. 'Roswell Park Comprehensive Cancer Insti, Buffalo, NY, USA; ${ }^{2}$ J. Graham Brown Cancer Center, Louisville, KY, USA; ${ }^{3}$ The University of North Carolina at Chapel Hill, NC, USA; ${ }^{4}$ Mayo Clinic Scottsdale School of Medicine, Tucson, AZ, USA; ${ }^{5}$ University of lowa Hospitals and Clinics, lowa City, IA, USA; ${ }^{6}$ University of California Los Angeles Sch, Porter Ranch, CA, USA; ${ }^{7}$ The Angeles Clinic and Research Institute, LoS Angeles, CA, USA; ${ }^{8}$ MD Anderson Cancer Center, Houston, TX, USA; ${ }^{9}$ Mt Sinai School of Medicine, New York, NY, USA; ${ }^{10}$ University Hospital Tuebingen, Tuebingen, Germany;

${ }^{11}$ Indiana University Simon Cancer Center, Indianapolis, IN, USA; ${ }^{12}$ University of Kiel, Kiel, Germany; ${ }^{13}$ Ap-hp Dermatology Cic Departments, Paris, France; ${ }^{14}$ Amgen Inc., Thousand Oaks, CA, USA; ${ }^{15}$ Rutgers Cancer Institute of New Jersey, New York, NY, USA

Background This is the first randomized trial evaluating an oncolytic virus with an immune checkpoint inhibitor in 


\section{University Library}

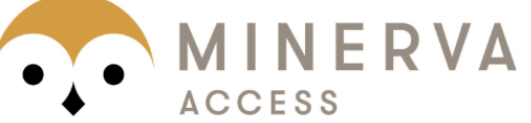

A gateway to Melbourne's research publications

Minerva Access is the Institutional Repository of The University of Melbourne

Author/s:

Dummer, R;Gyorki, D;Hyngstrom, J;Berger, A;Conry, R;Demidov, L;Chan, E;Radcliffe, H-

S;Faries, M;Ross, M

Title:

3-YEAR RESULTS OF THE PHASE 2 RANDOMIZED TRIAL FOR TALIMOGENE LAHERPAREPVEC (T-VEC) NEOADJUVANT TREATMENT PLUS SURGERY VS SURGERY IN PATIENTS WITH RESECTABLE STAGE IIIB-IVM1A MELANOMA

Date:

2020-11-01

Citation:

Dummer, R., Gyorki, D., Hyngstrom, J., Berger, A., Conry, R., Demidov, L., Chan, E., Radcliffe, H. -S., Faries, M. \& Ross, M. (2020). 3-YEAR RESULTS OF THE PHASE 2 RANDOMIZED TRIAL FOR TALIMOGENE LAHERPAREPVEC (T-VEC) NEOADJUVANT

TREATMENT PLUS SURGERY VS SURGERY IN PATIENTS WITH RESECTABLE STAGE IIBIVM1A MELANOMA. [Abstract]. JOURNAL FOR IMMUNOTHERAPY OF CANCER, 8 (Suppl 3), pp.A263-A263. https://doi.org/10.1136/jitc-2020-SITC2020.0432.

Persistent Link:

http://hdl.handle.net/11343/273971

License:

CC BY-NC-ND 